\title{
Cellular Cohomology in Homotopy Type Theory
}

\author{
Ulrik Buchholtz \\ Technische Universität Darmstadt \\ Darmstadt, Germany \\ buchholtz@mathematik.tu-darmstadt.de
}

\author{
Kuen-Bang Hou (Favonia) \\ Institute for Advanced Study \\ Princeton, NJ, USA \\ favonia@math.ias.edu
}

\begin{abstract}
We present a development of cellular cohomology in homotopy type theory. Cohomology associates to each space a sequence of abelian groups capturing part of its structure, and has the advantage over homotopy groups in that these abelian groups of many common spaces are easier to compute. Cellular cohomology is a special kind of cohomology designed for cell complexes: these are built in stages by attaching spheres of progressively higher dimension, and cellular cohomology defines the groups out of the combinatorial description of how spheres are attached. Our main result is that for finite cell complexes, a wide class of cohomology theories (including the ones defined through Eilenberg-MacLane spaces) can be calculated via cellular cohomology. This result was formalized in the Agda proof assistant.
\end{abstract}

\section{CCS CONCEPTS}

- Mathematics of computing $\rightarrow$ Lambda calculus; Algebraic topology; $•$ Theory of computation $\rightarrow$ Type structures; Constructive mathematics;

\section{KEYWORDS}

homotopy type theory, cellular cohomology, mechanized reasoning

\section{ACM Reference Format:}

Ulrik Buchholtz and Kuen-Bang Hou (Favonia). 2018. Cellular Cohomology in Homotopy Type Theory. In LICS '18: LICS '18: 33rd Annual ACM/IEEE Symposium on Logic in Computer Science, July 9-12, 2018, Oxford, United Kingdom. ACM, New York, NY, USA, 9 pages. https://doi.org/10.1145/3209108.3209188

\section{INTRODUCTION}

Homotopy type theory (HoTT) [27] is a new area exploring the potential of type-theoretic presentations of homotopy theory, an area originating from the study of topological spaces up to continuous transformation. It facilitates computer checking of proofs, and in some case results in the discovery of new proofs. (See for example [22].) Many proofs have since been mechanized and checked by proof assistants such as Agda [8], Lean [16] and Coq [6]. HoTT sheds new light on how type theory can be understood and leads to new type theories based on the resulting insights $[1-4,12,13]$.

Permission to make digital or hard copies of all or part of this work for personal or classroom use is granted without fee provided that copies are not made or distributed for profit or commercial advantage and that copies bear this notice and the full citation on the first page. Copyrights for components of this work owned by others than the author(s) must be honored. Abstracting with credit is permitted. To copy otherwise, or republish, to post on servers or to redistribute to lists, requires prior specific permission and/or a fee. Request permissions from permissions@acm.org.

LICS '18, Fuly 9-12, 2018, Oxford, United Kingdom

() 2018 Copyright held by the owner/author(s). Publication rights licensed to ACM.

ACM ISBN 978-1-4503-5583-4/18/07 ..\$15.00

https://doi.org/10.1145/3209108.3209188
One important concept in homotopy theory is homotopy groups, groups associated with every (pointed) space that reveals important structures of that space. However, it is difficult to calculate higher homotopy groups for many common spaces, for example spheres. As a result, homology and cohomology theories arise as an alternative way to study spaces.

The success of homotopy type theory begins with homotopy groups partially because of their simple type-theoretic definition. For example, the homotopy groups $\pi_{n}\left(\mathbb{S}^{n}\right)$ were calculated in [23]. Over time people have been extending the success in homotopy and cohomology theories as well. This paper is yet another milestone we have achieved.

The basic idea of cohomology theory is to study functions from cycles in a space. Functions from the cycles at a fixed dimension $n$ form a group, which is called the $n$th cohomology group. There are several ways to define a theory of such groups, some combinatorial and some axiomatic; amazingly, the classical theory states that the two approaches are essentially equivalent. Our goal is to recreate such an equivalence in HoTT.

In this paper we focus on a particular class of types, $C W$ complexes, which come with an explicit description of how the space is built by iterated attachment of disks. We will then introduce cellular cohomology theory, a combinatorial cohomology theory specifically defined for CW complexes. After that, we will define ordinary Eilenberg-Steenrod cohomology theory, an axiomatic framework for cohomology theory.

A large part of the text and Agda formulation presented in this paper already appeared in Hou (Favonia)'s PhD thesis [21]. However, one critical lemma, Lemma 7.3, was stated as a conjecture and the main theorem was only proved after the thesis. During the study, a critical component, degree, was radically changed to facilitate the proving.

\section{NOTATION}

We assume that readers are familiar with common type-theoretic expressions as in [21, 27]. In particular, we work in Martin-Löf type theory augmented with Voevodsky's univalence axiom and the pushout type constructor for homotopy pushouts. From these ingredients we can define the propositional [15] and the higher dimensional truncations [24]. Our constructions also work in the proposed cubical type theories [1-4, 12, 13].

We often use the word "space" as a synonym for "type" in order to facilitate the intuition of types as spaces. Pointed arrows are specially marked as $X \rightarrow Y$ for its importance in calculating degrees (Section 5). A special instance of pushouts that will occur throughout this paper is the homotopy cofiber of a pointed map 
$f: X \rightarrow Y$. It is defined to be the following pushout:

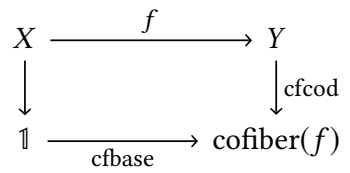

If the map $f$ is understood, we write $Y / X:=\operatorname{cofiber}(f)$.

\section{CW COMPLEXES}

A CW complex (also known as a cell complex) is an inductively defined type built by attaching cells, starting from points, lines, faces, and so on. The description consists of $A_{n}$ as the index set of cells at dimension $n$, along with functions $\alpha_{n}$ denoting how cells are attached. (We refer to [20, Chapter 0] for a discussion of CW complexes in a classical context.) With this combinatorial description at hand, one may define the cellular cohomology groups as shown in Section 4.

Many common types can be represented as CW complexes: the unit type, the spheres, the torus, and even the real projective spaces [10]. One can also build the two-cell complexes obtained by attaching a cell of dimension $m+1$ to a sphere of dimension $n$ with attaching map given by an element of $\pi_{m}\left(\mathbb{S}^{n}\right)$. A special case is that of the Moore spaces $M(\mathbb{Z} / q \mathbb{Z}, n)$ given by the element of $\pi_{n}\left(\mathbb{S}^{n}\right)$ corresponding to $q \in \mathbb{Z}$ under the isomorphism $\pi_{n}\left(\mathbb{S}^{n}\right) \simeq \mathbb{Z}$. The two-cell complexes are the simplest ones that are not spheres, and they form an important family of examples and counter-examples, see Section 5 below for an instance of this.

Let $X_{0}$ be the index set $A_{0}$ itself as the start and in general $X_{n}$ be the construction up to dimension $n .{ }^{1}$ A cell of index $a: A_{n+1}$ at dimension $n+1$ is specified by its boundary in $X_{n}$, denoted by the function $\alpha_{n+1}\langle a ;-\rangle$ from $\mathbb{S}^{n}$ to $X_{n}$. The type $X_{n+1}$ is then the result after attaching all cells at dimension $n+1$ to $X_{n}$. Formally, the function $\alpha_{n+1}$ is of type $A_{n+1} \times \mathbb{S}^{n} \rightarrow X_{n}$ describing the boundary of each cell. Inductively, the type $X_{n+1}$ is defined to be the pushout $X_{n} \sqcup_{A_{n+1} \times \mathbb{S}^{n} ; \alpha_{n+1} \text {; fst }} A_{n+1}$ : (Note that every pushout in homotopy type theory is a homotopy pushout.)

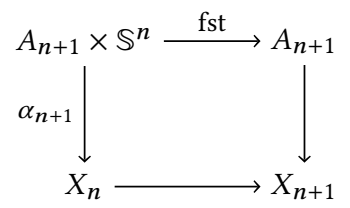

In this paper, we only work with finite $\mathrm{CW}$ complexes whose building process stops at some finite dimension and for which every $A_{n}$ is a finite set. Pictorially, a (finite) CW complex is the following iterated pushout, starting from the type $X_{0}: \equiv A_{0}$ and ending at some dimension.

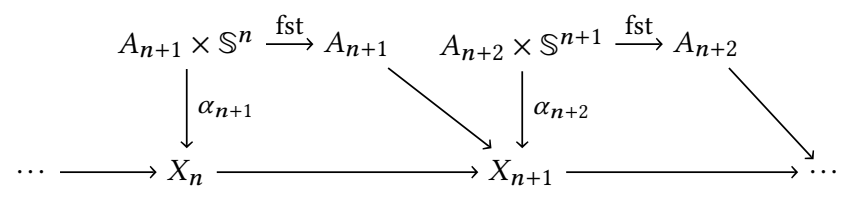

A pointed $\mathrm{CW}$ complex additionally requires $A_{0}$ to be pointed (and hence $X_{0}$ and all following pushouts).

\section{CELLULAR COHOMOLOGY}

Cohomology theory concerns functions from cycles, and one of the best ways to introduce it is through its dual, homology theory, which is about the cycles themselves. With access to an explicit, combinatorial description of a type, suitable algebraic structures can be defined for cycles.

To begin with, a one-dimensional cycle in homology theory (and cohomology theory) is a linear combination of oriented lines that at each any point has equally many incoming and outgoing lines counted with multiplicity (the in-degree equals the out-degree), see below for the precise definition. The intuition is to capture traversals in the space without worrying about the order of lines being traversed. Each coefficient in a cycle (as a linear combination) tracks the number of occurrences of a line, where a negative number signifies a traversal in the opposite direction. One can define the addition, subtraction and negation on these cycles as those on linear combinations.

Let $\tilde{\partial}_{1}$ be the function mapping a line from $a$ to $b$ to the linear combination $a-b$, which represents the oriented boundary of the line. Whether the in- and out- degrees are in balance reduces to whether the summation of $\tilde{\partial}_{1}$ of these lines is exactly zero. If we extend the function $\tilde{\partial}_{1}$ on lines to linear combinations of lines as $\partial_{1}$, then a linear combination of lines is a cycle if and only if it is in the kernel of $\partial_{1}$. In the context of CW complexes introduced in Section 3, the linear combinations of lines and points are the free abelian groups $\mathbb{Z}\left[A_{1}\right]$ and $\mathbb{Z}\left[A_{0}\right]$, respectively:

$$
\mathbb{Z}\left[A_{1}\right] \stackrel{\partial_{1}}{\longrightarrow} \mathbb{Z}\left[A_{0}\right]
$$

We would like to identify cycles up to cells at higher dimensions; in particular, if there is a two-dimensional cell filling the difference between two cycles (which is itself a cycle), then those two cycles should be regarded as the same. Intuitively, a cell fills a cycle if its boundary matches the cycle. Similar to $\partial_{1}$, one can define the boundary function $\partial_{2}$ for any two-dimensional cell $a: A_{2}$ by summing up lines traversed by $\alpha_{2}\langle a ;-\rangle$. A cycle can be filled if and only if it is in the image of $\partial_{2}$. Consider the following diagram:

$$
\mathbb{Z}\left[A_{2}\right] \stackrel{\partial_{2}}{\longrightarrow} \mathbb{Z}\left[A_{1}\right] \stackrel{\partial_{1}}{\longrightarrow} \mathbb{Z}\left[A_{0}\right]
$$

The subject of our interest, cycles up to identifications, is exactly the quotient of cycles (the kernel of $\partial_{1}$ ) by boundaries of cells at the next dimension (the image of $\partial_{2}$ ). This quotient forms the first cellular homology group of type $X$ (with integer coefficients), and groups for higher dimensions can be defined in a similar way. How exactly the boundary functions $\partial_{n}$ at higher dimensions should be defined from $A_{n}$ and $\alpha_{n}$ will be discussed later.

The sequence formed by the free abelian groups $\mathbb{Z}\left[A_{n}\right]$ and boundary maps $\partial_{n}$ is a chain complex. Cellular cohomology takes the dual of the sequence before calculating the quotients of kernels by images; it applies the contravariant functor hom $(-, G)$ for some

\footnotetext{
${ }^{1}$ This definition was adapted from Buchholtz's work in LEAN [9].
} 
given abelian group $G$ to the entire sequence. The dualized sequence is a cochain complex. ${ }^{2}$ The resulting diagram is

$$
\begin{aligned}
& \operatorname{hom}\left(\partial_{2}, G\right) \quad \operatorname{hom}\left(\partial_{1}, G\right) \\
& \operatorname{hom}\left(\mathbb{Z}\left[A_{2}\right], G\right) \longleftarrow \operatorname{hom}\left(\mathbb{Z}\left[A_{1}\right], G\right) \longleftarrow \operatorname{hom}\left(\mathbb{Z}\left[A_{0}\right], G\right)
\end{aligned}
$$

and the first cohomology group, denoted $H^{1}(X ; G)$, is the quotient of the kernel of $\operatorname{hom}\left(\partial_{2}, G\right)$ by the image of hom $\left(\partial_{1}, G\right)$. Groups at higher dimensions are defined in a similar way; we write $H^{n}(X ; G)$ as the $n$th cellular cohomology group with coefficients in $G$.

Boundary functions. One reasonable definition of $\partial_{n+1}$ on a cell $b$ at dimension $n+1$ is to individually calculate the coefficient $\operatorname{coeff}(b, a): \mathbb{Z}$ of each cell $a$ within the boundary of the cell $b$; that is, the boundary function is of the following form (where the $\sum$ below is the summation in linear algebra, not sum types):

$$
\partial_{n+1}(b): \equiv \sum_{a: A_{n}} \operatorname{coeff}(b, a) a .
$$

In order to make summation over a possibly infinite $A_{n}$ possible, it seems we have to assume $\operatorname{coeff}(b,-)$ always has finite support; this corresponds to the closure-finiteness condition in the classical theory, which is part of the definition of CW complexes and in fact what the "C" in the "CW" stands for. The classical condition says the boundary of each cell should be covered by a finite union of cells at lower dimensions, and so our assumption is well-motivated and may be necessary.

Intuitively, the value coeff $(b, a)$ should capture the number of (signed) occurrences of $a$ in the boundary of $b$. Considering the CW complex

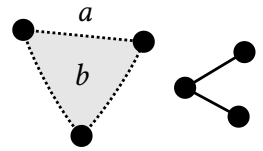

with a two-cell $b$ with boundary consisting of the line $a$ and two other lines, the value coeff $(b, a)$ should be 1 under suitable orientation. The trick is to identify all points and obtain a rose

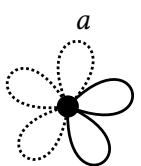

where the boundary of $b$ is now composed of loops at the center; the winding number of the loop $a$ is then the coefficient we are looking for. More precisely, the coefficient is the winding number of the function depicted in Figure 1, or more formally,

$$
\mathbb{S}^{1} \stackrel{\alpha_{2}\langle b ;-\rangle}{\longrightarrow} X_{1} \stackrel{\text { cfcod }}{\longrightarrow} X_{1} / X_{0} \simeq \bigvee_{-}: A_{1} \mathbb{S}^{1} \stackrel{\operatorname{proj}_{a}}{\longrightarrow} \mathbb{S}^{1}
$$

where $X_{1} / X_{0}$ is the cofiber of the inclusion from $X_{0}$ to $X_{1}$ and $\operatorname{proj}_{a}$ kills every loop except the one indexed by $a$. The projection function $\operatorname{proj}_{a}$ is definable whenever the index type has decidable

\footnotetext{
${ }^{2}$ The kernel-image quotienting is called homology in the classical literature, so the homology of a chain complex is homology, and the homology of a cochain complex is cohomology.
}

equality. For arbitrary dimension $n \geq 1$, we follow the same pattern to obtain a function from $\mathbb{S}^{n}$ to $\mathbb{S}^{n}$,

$$
\mathbb{S}^{n} \stackrel{\alpha_{n+1}\langle b ;-\rangle}{\longrightarrow} X_{n} \stackrel{\text { cfcod }}{\longrightarrow} X_{n} / X_{n-1} \simeq \bigvee_{-}: A_{n} \mathbb{S}^{n} \stackrel{\operatorname{proj}_{a}}{\longrightarrow} \mathbb{S}^{n}
$$

and then inspect its generalized winding number, namely its degree that will be defined in Section 5. The coefficient is defined as

$$
\operatorname{coeff}(b, a): \equiv \operatorname{deg}\left(\operatorname{proj}_{a} \circ e \circ \operatorname{cfcod} \circ \lambda x . \alpha_{n+1}\langle b ; x\rangle\right)
$$

where $e$ is the equivalence between $X_{n} / X_{n-1}$ and $\bigvee: A_{n} \mathbb{S}^{n}$. This finishes our definition of boundary functions in HoTT.

Reduced cellular cohomology theory. The cellular cohomology theory that is relevant in our paper is the reduced cellular cohomology theory for pointed types. A characteristic difference is that a reduced theory will assign the trivial group as the zeroth cohomology group of the unit type-the most trivial pointed type-rather than the group $\mathbb{Z}$; it is more stylish to have trivial groups for trivial types. To achieve this, the reduced homology theory augments the chain complex with $\epsilon$ to $\mathbb{Z}$ to give

$$
\cdots \longrightarrow \mathbb{Z}\left[A_{1}\right] \stackrel{\partial_{1}}{\longrightarrow} \mathbb{Z}\left[A_{0}\right] \stackrel{\epsilon}{\longrightarrow} \mathbb{Z}
$$

where $\epsilon$ sums up integer coefficients in $\mathbb{Z}\left[A_{0}\right]$; its dual,

$$
\cdots \longleftarrow \operatorname{hom}\left(\mathbb{Z}\left[A_{1}\right], G\right) \stackrel{\operatorname{hom}\left(\partial_{1}, G\right)}{\longleftarrow} \operatorname{hom}\left(\mathbb{Z}\left[A_{0}\right], G\right) \stackrel{\operatorname{hom}(\epsilon, G)}{\longleftarrow} \operatorname{hom}(\mathbb{Z}, G)
$$

leads to reduced cohomology groups. The ending $\mathbb{Z}$ effectively kills one degree of freedom in $\mathbb{Z}\left[A_{0}\right]$. In general, the reduced and unreduced integral homology groups only differ by a $\mathbb{Z}$ at the zeroth dimension.

\section{DEGREES}

The notion of the degree of a map of spheres of equal dimension is the key tool for defining and computing with cellular cohomology (and homology), as shown in the previous section. We therefore need a definition of degrees in HoTT.

The requirements of the definition are that we get for each $n: \mathbb{N}$ a function deg $:\left(\mathbb{S}^{n} \rightarrow \mathbb{S}^{n}\right) \rightarrow \mathbb{Z}$ satisfying at least the following properties:

(1) $\operatorname{deg}(\mathrm{id})=1$, where id : $\mathbb{S}^{n} \rightarrow \mathbb{S}^{n}$ is the identity function,

(2) $\operatorname{deg}(g \circ f)=\operatorname{deg}(g) \operatorname{deg}(f)$, where $g \circ f$ is the composition of $f$ and $g$,

(3) $\operatorname{deg}(\operatorname{susp}(f))=\operatorname{deg}(f)$, where $\operatorname{susp}(f): \mathbb{S}^{n+1} \rightarrow \mathbb{S}^{n+1}$ is the suspension of $f: \mathbb{S}^{n} \rightarrow \mathbb{S}^{n}$.

(4) $\operatorname{deg}(f)$ is the usual winding number of $f$, for $f: \mathbb{S}^{1} \rightarrow \mathbb{S}^{1}$. These requirements suffice to uniquely determine the degree function, although we have not formalized this fact.

One classical definition of degrees relies on homology theories, which are only now becoming available in HoTT [19], and their definition relies heavily on properties of the smash product, which is an unnecessary complication just for the definition of degrees.

Another approach is to apply the homotopy group functor to the function in question. However, for our purposes where we need good control on the interaction between the degree and a 


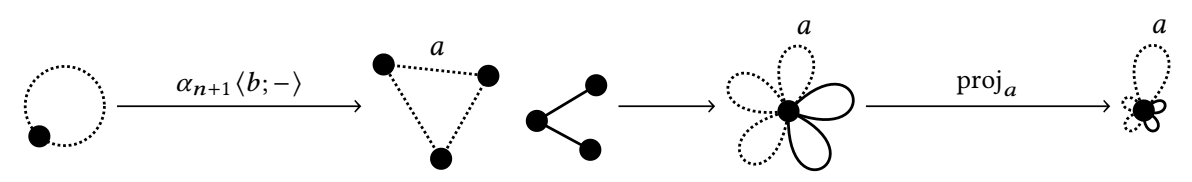

Figure 1: The function used to define $\operatorname{coeff}(b, a)$.

cohomology theory, we have found it expedient to use yet another approach. $^{3}$

The first observation is that by property (3) we can always reduce the computation of degree to the case of a pointed map. Another manifestation of this is that the canonical map from the set of pointed endo-arrows of the sphere $\left\|\mathbb{S}^{n} \rightarrow \mathbb{S}^{n}\right\|_{0}$ to the set of endoarrows $\left\|\mathbb{S}^{n} \rightarrow \mathbb{S}^{n}\right\|_{0}$ given by forgetting the point is an equivalence for $n \geq 1$. This means pointedness is free. For $n=0$, the identity on $\mathbb{S}^{0}(=2)$ has degree 1 , the map that swaps the points has degree -1 , while the remaining two maps have degree 0 , because they suspend to maps of the circle with winding number zero.

The second idea is to observe that the degree function is for each $n \geq 1$ a bijection on connected components. Since $\mathbb{Z}$ is a group, this suggests that we should be able to endow the type of pointed endoarrows of $\mathbb{S}^{n}$ with a natural group structure (up to homotopy). Thus, we want to define a group-like H-space structure on $\mathbb{S}^{n} \cdot \rightarrow \mathbb{S}^{n}$, such that the degree map becomes a group isomorphism.

A co- $\mathrm{H}$-space is the notion dual to that of an $\mathrm{H}$-space. This is a natural notion in homotopy theory, see [5] for a survey in the classical setting.

Definition 5.1. A co-H-space is a pointed type $A$ together with a comultiplication map $\sigma: A \rightarrow A \vee A$, and witnesses for the left and right counit laws, stating that $\pi_{1} \circ \sigma$ and $\pi_{2} \circ \sigma$ are homotopic as pointed maps to id $: A \rightarrow A$, where $\pi_{i}: A \vee A \rightarrow A$ denotes the projection on the left or right summand, for $i=1,2$ respectively.

This definition ensures that the type of pointed maps from a co-H-space to any pointed type is an $\mathrm{H}$-space.

Definition 5.2. A cogroup is a (homotopy) associative co-H-space together with a left inverse map $\lambda: A \rightarrow A$ such that $\nabla \circ(\lambda \vee$ id $) \circ \sigma$ is homotopic to the constant pointed map, where $\nabla: A \vee A \rightarrow A$ is the folding map, which is the identity on each summand.

Our main interest in cogroups stems from the following fact.

Proposition 5.3. Any suspension $\operatorname{susp}(A)$ has the structure of a cogroup with comultiplication given by the pinch map $\sigma: \operatorname{susp}(A) \rightarrow$ $\operatorname{susp}(A) \vee \operatorname{susp}(A)$.

The proof is easy and we omit it here. We note that there are cogroups that are not suspensions, for example the two-cell complex corresponding to an element of order 3 in $\pi_{34}\left(\mathbb{S}^{5}\right)$, see [7].

The next fact is the main result we need.

Proposition 5.4. If $X$ is a cogroup and $Y$ is a pointed type, then the set $\|X \rightarrow Y\|_{0}$ has a natural group structure.

${ }^{3}$ In Hou (Favonia)'s thesis [21], the degree was defined using the homotopy group functor.
Having thus equipped each type $\mathbb{S}^{n} \rightarrow \mathbb{S}^{n}$ for $n \geq 1$ with the structure of a group up to homotopy, it remains to actually define the degree functions.

For $n=1$ we must choose the winding number per property (4). Since we want property (3) to hold, we use the fact that the action of suspension on pointed maps, $(X \rightarrow Y) \rightarrow(\operatorname{susp}(X) \rightarrow \operatorname{susp}(Y))$ is a group homomorphism whenever $X$ is a cogroup. So it remains to verify that this map is an group isomorphism in the case of endo-arrows on $\mathbb{S}^{n}$, for $n \geq 1$. This follows from the Freudenthal suspension theorem [27, Theorem 8.6.4], in the same way that we prove that $\pi_{n}\left(\mathbb{S}^{n}\right) \simeq \mathbb{Z}$. In fact, the loop-suspension adjunction refines to a group isomorphism $(\operatorname{susp}(X) \rightarrow Y) \simeq(X \rightarrow \Omega Y)$, using the cogroup structure on $\operatorname{susp}(X)$ and the $(\mathrm{H}-)$ group structure on $\Omega Y$.

\section{EILENBERG-STEENROD COHOMOLOGY}

Unlike the above explicit construction, there is also an axiomatic framework for cohomology. Participants to the special year at Institute for Advanced Study have brought into HoTT the standard abstract framework for cohomology theories-Eilenberg-Steenrod axioms $[11,17,25]$. An (ordinary) ${ }^{4}$ reduced cohomology theory in HoTT may be defined as a contravariant functor $h$ from pointed types to sequences of abelian groups satisfying the following axioms. We write $h^{n}(X)$ to denote the $n$th group in the sequence for a pointed type $X$.

Before presenting these axioms, however, we need to define what it means to satisfy set-level axiom of choice, a condition stating that $\prod$ quantifiers and 0 -truncation commute. This will be used in one of the cohomology axioms shown later.

Definition 6.1 (set-level axiom of choice). A type A satisfies the set-level axiom of choice if, for any family of types $B$ indexed by $A$, the canonical function from $\left\|\prod_{a: A} B(a)\right\|_{0}$ to $\prod_{a: A}\|B(a)\|_{0}$ is an equivalence.

See [27] for more discussion about the axiom of choice and [25] for its role in cohomology theory in HoTT. Essentially, one could present the Eilenberg-Steenrod axioms without the axiom of choice, but it would be difficult for pointed arrows whose codomains are Eilenberg-Mac Lane spaces, an important example of cohomology theories, to satisfy these axioms within HoTT. In any case, because we only deal with cell complexes with finite cell sets, we do not have to worry about the axiom of choice, for it is provable in HoTT for finite sets.

In any case, here are the axioms we use:

${ }^{4}$ A cohomology theory is ordinary if it satisfies the last Eilenberg-Steenrod axiom. See below. 
Suspension. There is an isomorphism between $h^{n+1}(\operatorname{susp}(X))$ and $h^{n}(X)$, and the choice of isomorphisms is natural in $X$.

Exactness. For any pointed arrow $f: X \rightarrow Y$, the following sequence is exact, which means the kernel of $h^{n}(f)$ is exactly the image of $h^{n}$ (cfcod).

$h^{n}(\operatorname{cofiber}(f)) \stackrel{h^{n}(\mathrm{cfcod})}{\longrightarrow} h^{n}(Y) \stackrel{h^{n}(f)}{\longrightarrow} h^{n}(X)$

Wedge. Let $I$ be a type satisfying the set-level axiom of choice. For any family of pointed types $X$ indexed by $I$, the group morphism

$$
\iota^{*}: h^{n}\left(\bigvee_{i: I} X(i)\right) \rightarrow \prod_{i: I} h^{n}\left(X_{i}\right)
$$

induced by inclusions $X(i) \rightarrow \bigvee_{i: I} X(i)$ is a group isomorphism.

Dimension. For any integer $n \neq 0$, the group $h^{n}(2)$ is trivial.

The word ordinary refers to satisfying the dimension axiom. Interesting examples violating this axiom (but satisfying the rest), such as $K$-theories or complex cobordism, were discovered after the introduction of the framework, and are called extraordinary cohomology theories. Our result only handles ordinary ones.

It will be shown that these axioms uniquely identify cohomology groups for finite CW complexes. To begin with, we can calculate all the groups of the spheres directly from the axioms:

LeMma 6.2. For any $m, n: \mathbb{Z}$ such that $n \geq 1, h^{m}\left(\mathbb{S}^{n}\right)$ is isomorphic to $h^{0}(2)$ if $m=n$ and trivial otherwise.

Proof. Because the spheres are iterated suspensions of 2 , one can apply the suspension axiom till it reaches 2 and then the dimension axiom if the dimensions mismatch.

And similarly the groups for the bouquets, or wedges of spheres. They play an important role in our calculation of the groups of the CW complexes.

Lemma 6.3. For any $m, n: \mathbb{Z}$ such that $n \geq 0$ and any finite set $A, h^{m}\left(\bigvee_{-}: A \mathbb{S}^{n}\right)$ is isomorphic to $\prod_{-}: A h^{0}(2)$ if $m=n$ and trivial otherwise.

There is also an important consequence from the suspension and the exactness axioms which will be applied repeatedly:

Lemma 6.4. For any pointed arrow $f: X \rightarrow Y$, there exist a natural choice of $\gamma_{n}$ such that the following is a long exact sequence:
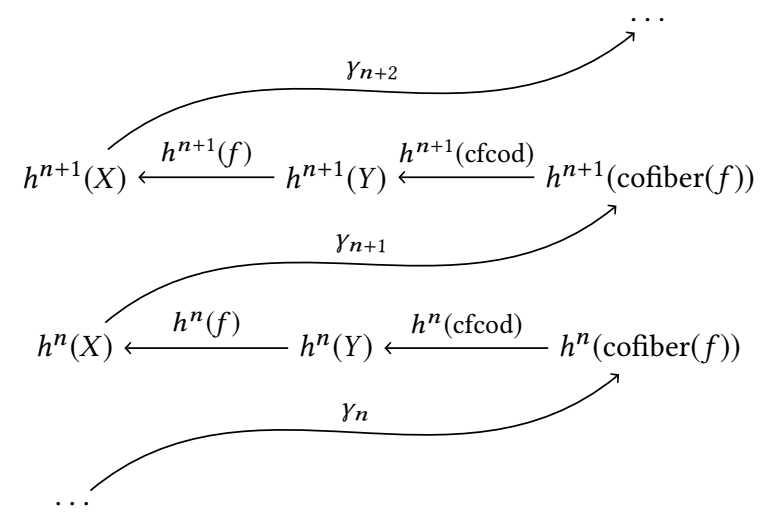

Proof. The key observation is that the cofiber of $\operatorname{cfcod}: Y \cdot \rightarrow$ cofiber $(f)$ is equivalent to $\operatorname{susp}(X)$, and thus $h^{n+1}$ on iterated cfcod's is equivalent to $h^{n+1}(\operatorname{susp}(\cdots))$, which by the suspension axiom is isomorphic to $h^{n}(\cdots)$. The exactness of $h^{n+1}$ on iterated cfcod's is given by the exactness axiom.

Finally, let us state the connection between cogroups and cohomology theories:

Proposition 6.5. Let $X$ be a cogroup, and $Y$ a pointed type. If $h$ is a cohomology theory, then the map

$$
h^{n}:\|X \cdot \rightarrow Y\|_{0} \rightarrow \operatorname{hom}\left(h^{n}(Y), h^{n}(X)\right)
$$

is a group homomorphism for each $n: \mathbb{Z}$.

Proof. This follows from the commutativity of the diagram:

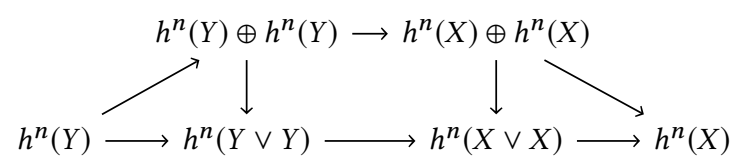

This key fact is a generalization of [20, Lemma 4.60] to cogroups, and will be used in the proof of Lemma 7.3.

\section{EQUIVALENCE OF TWO COHOMOLOGY THEORIES}

Our main result is the following:

THEOREM 7.1. For any ordinary reduced cohomology theory h, any pointed finite $C W$ complex $X$ and any $n: \mathbb{Z}, h^{n}(X)$ is isomorphic to $H^{n}\left(X ; h^{0}(2)\right)$.

This basically states that, on CW complexes, two notions of cohomology coincide. The significance is that it connects an explicit construction with a rather abstract framework over a wide range of types. Our approach is to break this theorem into two parts:

(1) We prove that from any ordinary cohomology theory $h$ satisfying the axioms, we may reconstruct a cochain complex so that its kernel-image quotients are the same as the groups directly given by $h$.

(2) We show that the reconstructed cochain complex is equivalent to the cochain complex used in the cellular cohomology, and thus the cellular cohomology groups and $h$ should agree.

In details, these steps are:

LEMMA 7.2 (REFORMULATION OF ORDINARY COHOMOLOGY GROUPS). For any ordinary reduced cohomology theory $h$ and any pointed finite $C W$ complex $X$, there is a choice of coboundary functions $\delta_{n}$ forming a cochain complex

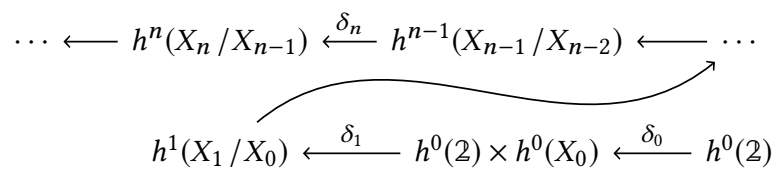

such that $h^{n}(X)$ is isomorphic to the quotient of the kernel of $\delta_{n+1}$ by the image of $\delta_{n}$ for any $n \geq 0$. 
The above lemma states that any ordinary cohomology groups are also the kernel-image quotients of some cochain complex, similar to cellular cohomology groups. It is then sufficient to show that the cochain complexes are equivalent.

LEMMA 7.3 (TWO COCHAIN COMPLEXES AGREE). Let $h$ be an ordinary reduced cohomology theory and $X$ be a pointed finite $C W$ complex. Let $\delta_{n}$ be the group homomorphisms given by Lemma 7.2 after reformulation. There exist an isomorphism

$$
k_{n}: h^{n}\left(X_{n} / X_{n-1}\right) \simeq \operatorname{hom}\left(\mathbb{Z}\left[A_{n}\right], h^{0}(2)\right)
$$

for any $n \geq 1$, an isomorphism for the zeroth dimension

$$
k_{0}: h^{0}(2) \times h^{0}\left(X_{0}\right) \simeq \operatorname{hom}\left(\mathbb{Z}\left[A_{0}\right], h^{0}(2)\right)
$$

and an isomorphism for the augmented part

$$
k_{-1}: h^{0}(2) \simeq \operatorname{hom}\left(\mathbb{Z}, h^{0}(2)\right)
$$

such that for any $n \geq 1$, the following square commutes

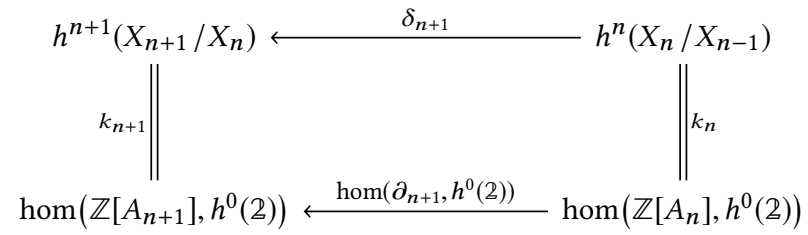

and similarly for the case $n=0$ and the augmented part with suitable groups and group homomorphisms.

Our main result immediately follow from these two lemmas.

Proof of Theorem 7.1. Let $\tilde{h}^{n}(X)$ be the kernel-image quotients of the coboundary maps $\delta$ given by Lemma 7.2. We have $\tilde{h}^{n}(X) \simeq$ $h^{n}(X)$ by the lemma. Because the coboundary maps $\delta$ and the dualized boundary maps are equivalent according to Lemma 7.3 , the resulting groups $\tilde{h}^{n}(X)$ and $H^{n}\left(X ; h^{0}(2)\right)$ are also isomorphic, and thus the theorem.

In the following subsections, we will sketch the proofs of Lemmas 7.2 and 7.3.

\subsection{Reformulation of Cohomology Groups}

The central idea is to construct as many cofibers as possible from its cellular description, and then apply the exactness axiom on these cofibers to obtain long exact sequences by Lemma 6.4. From the obtained long exact sequences we can then calculate the groups of our interest.

Before constructing those cofibers, it is essential to observe that there is a lemma complimentary to Lemma 7.3:

LEMMA 7.4. For any $m \neq n: \mathbb{Z}$ such that $n \geq 1, h^{m}\left(X_{n} / X_{n-1}\right)$ is trivial. Moreover, for any $m: \mathbb{Z}$ such that $m \neq 0, h^{m}\left(X_{0}\right)$ is also trivial.

Proof Sкetch. Let $a$ be the distinguished point of $X$. It is sufficient to show $X_{n} / X_{n-1} \simeq V_{-}: A_{n} \mathbb{S}^{n}$ and $X_{0} \simeq \bigvee_{\sum_{x: A_{0}}(a \neq x)^{2}}$ because the rest follows from Lemma 6.3. The intuition is that, if we shrink $X_{n-1}$ into a point, the remaining of $X_{n}$ is the attachment of $A_{n}$ many cells at the $n$th dimension, which results into a wedge of $\mathbb{S}^{n}$ indexed by $A_{n}$. The zeroth dimension is special because one of the points-which is the distinguished point $a$ here-is selected as the center of the wedge.

The way to construct numerous cofiber squares is to consider the following grid diagram where every grid is a pushout square.

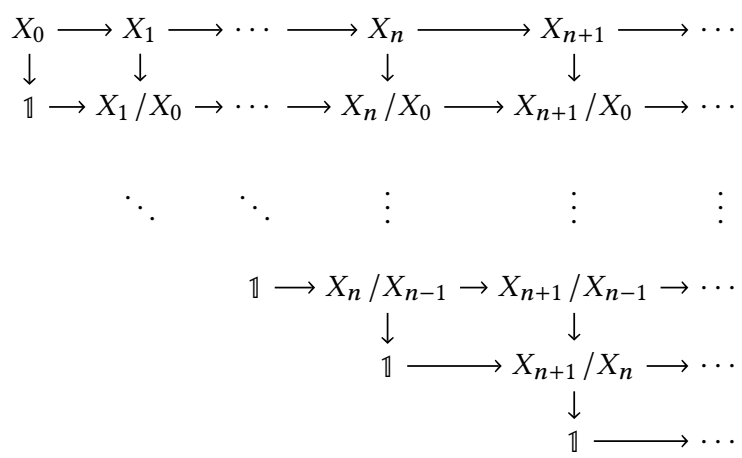

Any square (consisting of one or more grids) having the unit type $\mathbb{1}$ at the bottom left is a cofiber square and generates a long exact sequence by Lemma 6.4. Three conclusions can be drawn by choosing different cofiber squares:

First, we can zoom in on a grid on the diagonal:

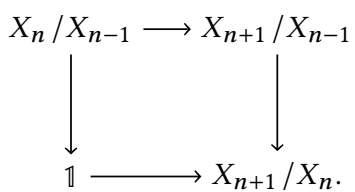

Through Lemma 6.4, this grid generates the following exact sequence:

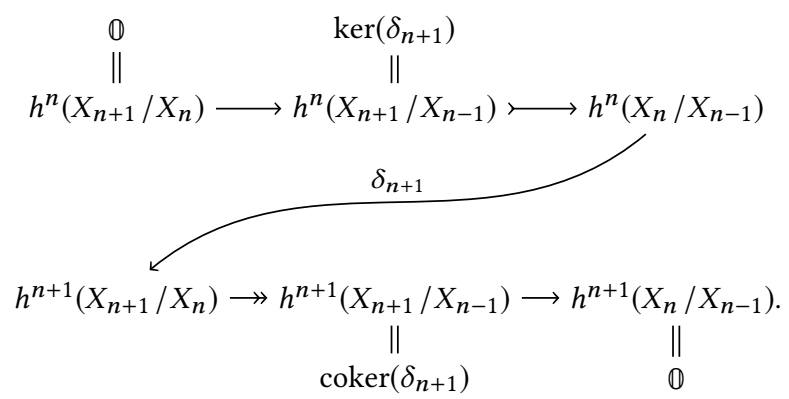

We choose the coboundary function $\delta_{n+1}$ to be the middle function as required. Because $h^{n}\left(X_{n+1} / X_{n}\right)$ is trivial, from the exactness we know $h^{n}\left(X_{n+1} / X_{n-1}\right)$ is isomorphic to the kernel of $\delta_{n+1}$ and the group homomorphism from it is injective. Dually, we know $h^{n+1}\left(X_{n+1} / X_{n-1}\right)$ is isomorphic to the cokernel of $\delta_{n+1}$ and the group homomorphism to it is surjective.

Secondly, let's turn our focus to this square:

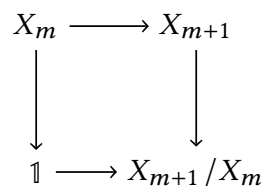


which by Lemma 6.4 gives this exact sequence:

$$
h^{n}\left(X_{m+1} / X_{m}\right) \rightarrow h^{n}\left(X_{m+1}\right) \rightarrow h^{n}\left(X_{m}\right) \rightarrow h^{n+1}\left(X_{m+1} / X_{m}\right)
$$

When $n \notin\{m, m+1\}$, both $h^{n}\left(X_{m+1} / X_{m}\right)$ and $h^{n+1}\left(X_{m+1} / X_{m}\right)$ are trivial by Lemma 7.4; therefore, by the exactness of the above sequence, $h^{n}\left(X_{m+1}\right) \simeq h^{n}\left(X_{m}\right)$. In other words, cells at dimensions much higher or much lower than $n$ are irrelevant to the cohomology group at dimension $n$. This implies that there are at most three different values of $h^{n}\left(X_{m}\right)$ up to isomorphism:

(1) $h^{n}\left(X_{n-1}\right) \simeq h^{n}\left(X_{n-2}\right) \simeq \cdots \simeq h^{n}\left(X_{0}\right) \simeq \mathbb{O}$. The intuition is that $X_{m}$ for any $m<n$ does not have any interesting information at dimension $n$.

(2) $h^{n}\left(X_{n}\right) . X_{n}$ has the cells at dimension $n$, but lacks the cells at dimension $(n+1)$ which may identify some cycles at dimension $n$.

(3) $h^{n}\left(X_{n+1}\right) \simeq h^{n}\left(X_{n+2}\right) \simeq \cdots \simeq h^{n}(X)$. Cells at dimension $(n+2)$ or above play no role in the $n$th cohomology group.

It is thus sufficient to study $h^{n}\left(X_{n+1}\right)$ for the $n$th cohomology group of $X$.

Finally, we investigate the square

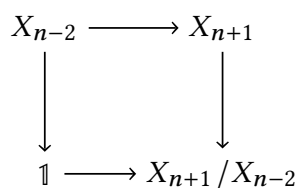

which generates the exact sequence

$$
h^{n-1}\left(X_{n-2}\right) \longrightarrow h^{n}\left(X_{n+1} / X_{n-2}\right) \longrightarrow h^{n}\left(X_{n+1}\right) \longrightarrow h^{n}\left(X_{n-2}\right)
$$

From the previous cofiber square we know both $h^{n-1}\left(X_{n-2}\right)$ and $h^{n}\left(X_{n-2}\right)$ are trivial, and again by the exactness $h^{n}\left(X_{n+1} / X_{n-2}\right) \simeq$ $h^{n}\left(X_{n+1}\right)$. Therefore, we have

$$
h^{n}\left(X_{n+1} / X_{n-2}\right) \simeq h^{n}\left(X_{n+1}\right) \simeq h^{n}\left(X_{n+2}\right) \simeq \cdots \simeq h^{n}(X) .
$$

This means it is sufficient to calculate $h^{n}\left(X_{n+1} / X_{n-2}\right)$.

Combining these three observations, we have the following commuting square for $n \geq 2$ :

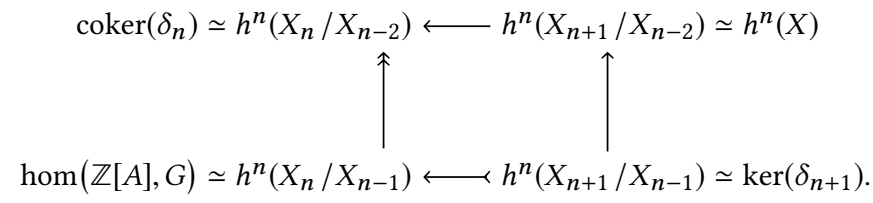

We can further infer that the top homomorphism is injective and the right one is surjective by applying Lemma 6.4 to the following two cofiber squares, respectively,
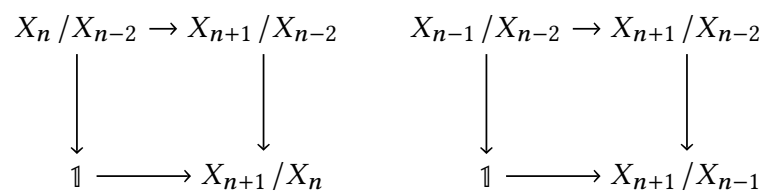

and finally obtain the desired isomorphism

$$
h^{n}(X) \simeq \operatorname{ker}\left(\delta_{n+1}\right) / \operatorname{im}\left(\delta_{n}\right)
$$

by the following lemma from group theory:

Lemma 7.5. Let $Q \subseteq P$ be two subgroups of $G$ where $Q$ is normal. If we have a group $K$ and a commuting diagram as follows, where the group homomorphism from $P$ to $G$ is the canonical inclusion and the one from $G$ to $G / Q$ is the quotienting, then $K \simeq P / Q$.

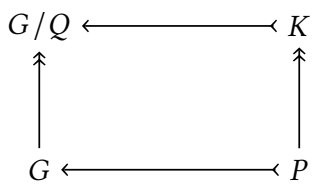

By choosing $P$ to be the kernel and $Q$ the image, we have the desired formula that $K$ is the quotient. ( $Q$ is normal because it is a subgroup of an abelian group.) This shows that for any $n \geq 2, h^{n}(X)$ is isomorphic to the kernel-image quotient of adjacent coboundary functions $\delta_{n}$. The cases for $n=0$ or 1 can also be derived from the grid diagram similarly but with special care of the distinguished point of $X_{0}: \equiv A_{0}$ as demonstrated in the proof of Lemma 7.4. This concludes the proof of Lemma 7.2.

\subsection{Equivalence of Two Cochain Complexes}

Recall the isomorphisms and commuting squares we want:

$$
\begin{aligned}
k_{n}: h^{n}\left(X_{n} / X_{n-1}\right) & \simeq \operatorname{hom}\left(\mathbb{Z}\left[A_{n}\right], h^{0}(2)\right) \\
k_{0}: h^{0}(2) \times h^{0}\left(X_{0}\right) & \simeq \operatorname{hom}\left(\mathbb{Z}\left[A_{0}\right], h^{0}(2)\right) \\
k_{-1}: h^{0}(2) & \simeq \operatorname{hom}\left(\mathbb{Z}, h^{0}(2)\right)
\end{aligned}
$$

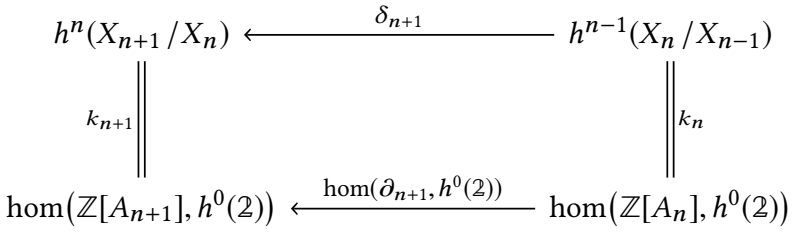

The isomorphisms $k_{n}$ for $n \geq 1$ arise from the type equivalences

$$
\begin{gathered}
X_{n} / X_{n-1} \simeq \bigvee_{: A_{n}} \mathbb{S}^{n} \\
X_{0} \simeq \bigvee_{\sum_{x: A_{0}}(a \neq x)}^{2} 2
\end{gathered}
$$

and then Lemma 6.3. The zeroth dimension needs special attention for the same reason given in the proof of Lemma 7.4: the point $a$ is used as the center of the wedge. Therefore, we have to add one copy of $h^{0}(2)$ to the left hand side to make ends meet. There is one more subtlety in $k_{0}$; here is a possible break down of the isomorphism:

$$
\begin{aligned}
k_{0}: h^{0}(2) \times h^{0}\left(X_{0}\right) \simeq h^{0}(2) & \times \prod_{\sum_{a: A_{0}} a \neq x} h^{0}(2) \\
\simeq & \prod_{A_{0}} h^{0}(2) \simeq \operatorname{hom}\left(\mathbb{Z}\left[A_{0}\right], h^{0}(2)\right)
\end{aligned}
$$

It would be naive to directly collect values arising from $h^{0}(2)$ and $h^{0}\left(X_{0}\right)$ (through $\left.\prod_{\sum_{a: A_{0}} a \neq x} h^{0}(2)\right)$ and form a function in the product group $\prod_{A_{0}} h^{0}(2)$. That unfortunately would not make all the needed squares commute. The correct way to merge an element $g$ 
from $h^{0}(2)$ with an element $f$ from $\prod_{\sum_{a: A_{0}} a \neq x} h^{0}(2)$ is to construct a function $h$ such that

$$
h(x)= \begin{cases}g & \text { if } a=x \\ f(x) \cdot g & \text { if } a \neq x .\end{cases}
$$

where the binary operation is the one specified by the group $h^{0}(2)$. The intuition is that in cohomology we care about the difference between values on points, not their absolute values; thus, the natural way to maintain the difference is to calculate the relative values when the center $a$ is chomped and restore them when the center $a$ revives. The first component from $h^{0}(2)$ in the isomorphism may be seen as the standard sea level for the second component.

Once the correct isomorphisms are in place, the commuting squares are relatively straightforward. The simplest proof we found is to rephrase the $\delta_{n}$ and the dualized $\partial_{n}$, through the isomorphisms $k_{n}$ and Proposition 6.5, as homomorphisms between the groups $\prod_{A_{n}} h^{0}(2)$ and $\prod_{A_{n+1}} h^{0}(2)$. When the sets $A_{n}$ are finite, all these maps can be rephrased as

$$
\lambda f . \lambda\left(x: A_{n+1}\right) . \sum_{y: A_{n}} f(y)^{\operatorname{deg}\left(\sigma_{x, y}\right)}
$$

for some endo-arrows $\sigma_{x, y}$ of the spheres. (Note that the $\sum$ here is the summation defined by the group structure, not the sigma types.) Therefore, it boils down to proving that the parameters $\sigma$ for the $\delta_{n}$ and the dualized $\partial_{n}$ have the same degrees. In our setting, the parameters for coboundary maps are always the suspensions of those for boundary maps, and thus share the same degree by the property 3 of the degree function. This concludes the proof of Lemma 7.3. The significance of the finiteness of $A_{n}$ is that it turns group products into direct sums and consequently gives an explicit description of the inverse map in the wedge axiom of the Eilenberg-Steenrod framework and enables summation over cells at a dimension.

\section{MECHANIZATION IN AGDA}

The main theorem (Theorem 7.1) and all lemmas have been mechanized in the proof assistant Agda, using the HoTT-Agda library [8]. The statement of the main theorem can be found at https:/github. com/HoTT/HoTT-Agda/blob/f46225f9428520572424a07fec71b5143f/ theorems/cw/cohomology/AxiomaticIsoCellular.agda\#L18-L25.

In order to mechanize the proofs in our work, significant improvements have been done to the general framework and it is difficult to quantify them. Already there are more than 4,000 lines of code written specifically for the work presented in this paper, among which much engineering was done to make it type-check within reasonable time and memory space.

\section{CONCLUSION AND FURTHER WORK}

The main theorem in this paper dealt with the case where every cell set $A_{n}$ was finite and the dimension of the complex is finite. A first, relatively straight-forward extension, is to consider infinitedimensional cell complexes with finite cell sets.

We believe that it should be possible to further generalize the result to (certain) infinite cell sets as follows. Recall that the spheres are $\omega$-compact: if $X: \omega \rightarrow \mathcal{U}$ is a sequential diagram of types, then any $f: \mathbb{S}^{n} \rightarrow \lim _{k} X_{k}$ factors through some $X_{k}$. More precisely:
Definition 9.1. A type $C$ is $\omega$-compact if for any sequential diagram $X: \omega \rightarrow \mathcal{U}$, the canonical map

$$
\lim _{\longrightarrow k}\left(C \rightarrow X_{k}\right) \rightarrow\left(C \rightarrow \lim _{\longrightarrow} X_{k}\right)
$$

is an equivalence.

CONJEcture 9.2. If each $A_{n}$ is either finite or isomorphic to $\mathbb{N}$, then each stage of a cell complex $X_{n}$ is a sequential colimit.

Depending on how precisely this is formalized, it is possible that countable choice is needed: there should be a natural map from a sequential colimit of finite subcomplexes into $X_{n}$, and $\omega$ compactness of spheres should ensure this is an equivalence.

Note that classically, almost all cell complexes we want to consider have explicitly given cell sets that are either finite or isomorphic to $\mathbb{N}$. It is also worth remarking that assuming countable choice may not interfere with a computational interpretation of type theory, since countable choice holds in realizability models of extensional type theory. (On the other hand, countable choice is not provable in homotopy type theory, as shown in [14].) We leave these deliberations for future work.

Another obvious line of inquiry is to use our setup to do cellular homology theory. Once a good library has been developed for homology theory, we in fact expect that the result corresponding to our Theorem 7.1 will be simpler in the homology setting.

We can obtain a direct comparison between our results concerning bare $\mathrm{CW}$ complexes, and results about usual $\mathrm{CW}$ complexes as topological spaces using the real-cohesive type theory of [26]. A CW complex in topological spaces corresponds to a CW complex in the sense of Section 3, but with all spheres replaced by their topological counterparts, viz., the unit spheres in $\mathbb{R}^{n+1}$. The shape functor will take such a topological $\mathrm{CW}$ complex to a CW complex in our sense, since it preserves colimits and maps the topological spheres to their homotopy types.

Eventually, it is also our hope that our work can be part of a full formalization of practical algorithms used in constructive algebraic topology, such as those in the Kenzo system [18].

\section{ACKNOWLEDGMENTS}

We want to acknowledge the valuable comments that we received on earlier drafts and presentations of the work in this paper from Carlo Angiuli, Guillaume Brunerie, Evan Cavallo, Floris van Doorn, Robert Harper, Egbert Rijke, and several anonymous reviewers.

This research was sponsored by the National Science Foundation under grant number DMS-1638352 and the Air Force Office of Scientific Research under grant number FA9550-15-1-0053. The authors would also like to thank the Isaac Newton Institute for Mathematical Sciences for its support and hospitality during the program "Big Proof" when part of work on this paper was undertaken; the program was supported by Engineering and Physical Sciences Research Council under grant number EP/K032208/1. The views and conclusions contained in this document are those of the authors and should not be interpreted as representing the official policies, either expressed or implied, of any sponsoring institution, government or any other entity. 


\section{REFERENCES}

[1] Carlo Angiuli and Robert Harper. 2016. Computational Higher Type Theory II Dependent Cubical Realizability. arXiv:cs.LO/1606.09638

[2] Carlo Angiuli, Robert Harper, and Todd Wilson. 2016. Computational Higher Type Theory I: Abstract Cubical Realizability. arXiv:cs.LO/1604.08873

[3] Carlo Angiuli, Robert Harper, and Todd Wilson. 2017. Computational Higherdimensional Type Theory. In Proceedings of the 44th ACM SIGPLAN Symposium on Principles of Programming Languages (POPL 2017). ACM, New York, NY, USA 680-693. https://doi.org/10.1145/3009837.3009861

[4] Carlo Angiuli, Kuen-Bang Hou (Favonia), and Robert Harper. 2017. Computational Higher Type Theory III: Univalent Universes and Exact Equality. arXiv:cs.LO/1712.01800

[5] Martin Arkowitz. 1995. Co- $H$-spaces. In Handbook of algebraic topology. NorthHolland, Amsterdam, 1143-1173. https://doi.org/10.1016/B978-044481779-2/ 50024-9

[6] Andrej Bauer, Jason Gross, Peter LeFanu Lumsdaine, Michael Shulman, Matthieu Sozeau, and Bas Spitters. 2017. The HoTT Library: A Formalization of Homotopy Type Theory in Coq. In Proceedings of the 6th ACM SIGPLAN Conference on Certified Programs and Proofs (CPP 2017). ACM, New York, NY, USA, 164-172. https://doi.org/10.1145/3018610.3018615

[7] Israel Berstein and John R. Harper. 1989. Cogroups which are not suspensions. In Algebraic topology, Proc. Int. Conf., Arcata/Calif. 1986 (LNM), Gunnar Carlsson, Ralph Cohen, Haynes Miller, and Douglas Ravenel (Eds.), Vol. 1370. Springer, Heidelberg, 63-86. https://doi.org/10.1007/BFb0085219

[8] Guillaume Brunerie, Kuen-Bang Hou (Favonia), Evan Cavallo, Eric Finster, Jesper Cockx, Christian Sattler, Chris Jeris, Michael Shulman, et al. 2011-2018. Homotopy Type Theory in Agda. https://github.com/HoTT/HoTT-Agda

[9] Ulrik Buchholtz. 2017. Cellular complexes in Lean. https://github.com/ leanprover/lean2/blob/master/hott/homotopy/cellcomplex.hlean

[10] Ulrik Buchholtz and Egbert Rijke. 2017. The real projective spaces in homotopy type theory. In 32nd Annual ACM/IEEE Symposium on Logic in Computer Science (LICS 2017). IEEE, New York, NY, USA, 1-8. https://doi.org/10.1109/LICS.2017. 8005146

[11] Evan Cavallo. 2015. Synthetic Cohomology in Homotopy Type Theory. Master's thesis. http://www.cs.cmu.edu/ ecavallo/works/thesis.pdf

[12] Evan Cavallo and Robert Harper. 2018. Computational Higher Type Theory IV: Inductive Types. arXiv:cs.LO/1801.01568

[13] Cyril Cohen, Thierry Coquand, Simon Huber, and Anders Mörtberg. 2016. Cubical Type Theory: a constructive interpretation of the univalence axiom. In 21st International Conference on Types for Proofs and Programs (TYPES 2015). Schloss Dagstuhl. Leibniz-Zent. Inform., Wadern. arXiv:1611.02108 To appear.

[14] Thierry Coquand, Bassel Mannaa, and Fabian Ruch. 2017. Stack semantics of type theory. In 32nd Annual ACM/IEEE Symposium on Logic in Computer Science (LICS 2017). IEEE, New York, NY, USA, 1-11. https://doi.org/10.1109/LICS.2017.8005130

[15] Floris van Doorn. 2016. Constructing the Propositional Truncation Using Nonrecursive HITs. In Proceedings of the 5th ACM SIGPLAN Conference on Certified Programs and Proofs (CPP 2016). ACM, New York, NY, USA, 122-129. https: //doi.org/10.1145/2854065.2854076

[16] Floris van Doorn, Jakob von Raumer, and Ulrik Buchholtz. 2017. Homotopy Type Theory in Lean. In Interactive Theorem Proving (ITP 2017). Springer, Cham, 479-495. https://doi.org/10.1007/978-3-319-66107-0_30

[17] Samuel Eilenberg and Norman E. Steenrod. 1945. Axiomatic Approach to Homology Theory. Proceedings of the National Academy of Sciences of the United States of America 31, 4 (1945), 117-120. http://www.pnas.org/content/31/4/117.full.pdf

[18] Julio Rubio Garcia, Francis Sergeraert, and Yvon Siret. 1998. Kenzo: A Symbolic Software for Effective Homology Computation. Retrieved Jan. 30, 2018 from https://www-fourier.ujf-grenoble.fr/ sergerar/Kenzo/

[19] Robert Graham. 2017. Synthetic Homology in Homotopy Type Theory. arXiv: 1706.01540

[20] Allen Hatcher. 2002. Algebraic Topology. Cambridge University Press, Cambridge, UK. xii+544 pages. https://www.math.cornell.edu/ hatcher/AT/ATpage.html

[21] Kuen-Bang Hou (Favonia). 2017. Higher-Dimensional Types in the Mechanization of Homotopy Theory. Ph.D. Dissertation. Carnegie Mellon University. http: //favonia.org/thesis

[22] Kuen-Bang Hou (Favonia), Eric Finster, Daniel R. Licata, and Peter LeFanu Lums daine. 2016. A Mechanization of the Blakers-Massey Connectivity Theorem in Homotopy Type Theory. In Proceedings of the 31st Annual IEEE Symposium on Logic in Computer Science (LICS 2016). IEEE, New York, NY, USA, 565-574. https://doi.org/10.1145/2933575.2934545

[23] Daniel R. Licata and Guillaume Brunerie. 2013. $\pi_{n}\left(\mathbb{S}^{n}\right)$ in Homotopy Type Theory. In Proceedings of the 3rd International Conference on Certified Programs and Proofs (CPP 2013), Georges Gonthier and Michael Norrish (Eds.). Springer International Publishing, Cham, 1-16. https://doi.org/10.1007/978-3-319-03545-1_1

[24] Egbert Rijke. 2017. The join construction. arXiv:1701.07538 Preprint.

[25] Michael Shulman. 2013. Cohomology. Retrieved Jan. 30, 2018 from http: //homotopytypetheory.org/2013/07/24/cohomology/
[26] Michael Shulman. 2017. Brouwer's fixed-point theorem in real-cohesive homotopy type theory. Mathematical Structures in Computer Science (2017), 1-86. https://doi.org/10.1017/S0960129517000147

[27] The Univalent Foundations Program. 2013. Homotopy Type Theory: Univalent Foundations of Mathematics. Institute for Advanced Study. arXiv:1308.0729 http://homotopytypetheory.org/book 